\title{
X Works Cited
}

\section{X.1 Primary Sources}

Arenas, Reinaldo. La loma del Angel. Malaga: Dador, Traición, 1986.

Bentzon, Thérèse. Yette. Histoire d'une jeune Créole. [Reprint of Paris: J. Hetzel, 1880 edition.] In one volume with J. Levilloux, Les créoles ou la Vie aux Antilles. Morne-Rouge: Éd. des Horizons Caraïbes, 1977, pp. 273-409.

Bergeaud, Émeric. Stella, translated by Adriana Umaña Hossman. N.p.: Markus Wiener, 2014. (Stella, edited by Beaubrun Ardouin. Paris: E. Dentu, 1859.)

Catineau-La-Roche, Pierre-Marie. Notice sur la Guyane française, suivie des motifs qui font désirer que la colonisation projetée sur la Mana soit dirigée par une association en concurrence avec le gouvernement. Paris: Imprimerie de Fain, 1822. Available online at http://gallica.bnf.fr/ark:/12148/bpt6k5460709r.r=Catineau-La-Roche.langEN. Accessed 23 June 2016.

Chapus, Eugène, Victor Charlier: Titime? Histoires de l'autre-monde. Paris: E. Renduel, 1833.

Chateaubriand, François-René de. Atala; René, translated by Rayner Heppenstall. Richmond: Oneworld Classics, 2010.

Chateaubriand, François-René de. Memoirs from beyond the Tomb, translated by Robert Baldick. London: Penguin Classics, 2014. (Mémoires d'outre-tombe [1848], edited by Maurice Levaillant. Paris: Gallimard, 1958.)

Chenêt, Jean-Baptiste. Études poétiques ou chants du barde glanés chez les muses. Paris: Imprimerie administrative de Paul Dupont, 1846.

Coicou, Massillon. Poésies nationales. Paris: Goupy et Jourdan, 1892.

Condé, Maryse. Crossing the Mangrove, translated by Richard Philcox. New York: Anchor Books, 1995. (Traversée de la Mangrove. Paris: Mercure de France, 1989.)

Condesa de Merlín, Maria de las Mercedes Santa Cruz y Montalvo. La Havane. 3 vols. Paris: Amyot, 1844.

Condesa de Merlín, María de las Mercedes Santa Cruz y Montalvo. "Les esclaves dans les colonies espagnoles." Revue des deux mondes, initial period, series 4, no. 26, 1841, pp. $734-769$.

Confiant, Raphaël. Adèle ou la pacotilleuse. Paris: Mercure de France, 2005.

Confiant, Raphaël. Le Nègre et l'Amiral. Paris: Grasset, 1988.

Constant, Benjamin. "Écrits d'un humaniste." Revue encyclopédique, 1825, p. 59.

Copans, Jean, and Jean Jamin, editors. Aux origines de l'anthropologie française. Les mémoires de la Soc. des Observateurs de l'Homme en l'An VIII, with a preface by Jean-Paul Faivre. Paris: Le Sycomore, 1978.

Coussin, J.H.J. Eugène de Cerceil ou les Caraïbes. 3 vols. Paris: Igonette, 1824.

D’Alaux, Gustave. "La littérature jaune.” Revue des deux mondes, new period, series 1. Part I: vol. XV, 1852, pp. 938-967; Part II: vol. XVI, 1852, pp. 1048-1085.

D’Alaux, Gustave. "Les mœurs et la littérature nègres." Revue des deux mondes, new period, series 1, vol. XIV, 1852, pp. 762-794.

del Monte, Domingo. "Letter of August 12, 1826." Centón epistolario de Domingo del Monte. Vol. 1. Havana, 1929.

Eyma, Louis-Xavier. “Les Borgias Noirs.” Les Peaux Noires. Paris: M. Lévy, 1857, pp. 109-132. Eyma, Louis-Xavier. Le roi des Tropiques. Paris: Michel Lévy Frères, 1860.

Ә OpenAccess. (c) 2018, Gesine Müller. (cc) BY-NC-ND This work is licensed under the Creative Commons Attribution-Non Commerical-NoDerivs 4.0 License. https://doi.org/10.1515/9783110495416-010 
Eyma, Louis-Xavier. La Vie aux Etats-Unis. Notes de voyage. Paris: Plon, 1876.

Galván, Manuel de Jesús. Enriquillo. Leyenda histórica dominicana (1503-1533) [1879]. Mexiko: Porrúa, 1976.

Galván, Manuel de Jesús. Enriquillo. Leyenda histórica dominicana (1503-1533) [1879]. Notas del autor. Santo Domingo: Corripio, 1990.

Glissant, Édouard. “À propos de Tout-Monde. Ein Gespräch mit Ralph Ludwig.” (Conversation with Ralph Ludwig.) Marie Galante, August 17, 1994.

Glissant, Édouard, and Patrick Chamoiseau. “De loin.” (Protest letter written in January 2005 by Édouard Glissant and Patrick Chamoiseau to then French minister of the interior Nicolas Sarkozy.) Potomitan. 18 May 2016. Available online at: http://www.potomitan. info/articles/deloin.php. Accessed 23 June 2016.

Gómez de Avellaneda, Gertrudis. Sab [1841], edited by José Servera. Madrid: Cátedra, 1997.

Gómez de Avellaneda, Gertrudis. Sab [1841], edited by Mary Cruz. La Habana: Ed. Arte y Literatura, 1976.

Gómez de Avellaneda, Gertrudis. Cartas inéditas y documentos. 1859 a 1864, illustrated collection edited by José Augusto Escoto. Matanzas: Imprenta La Pluma de Oro, 1912.

Granier de Cassagnac, Adolphe. Voyage aux Antilles françaises, anglaises, danoises, espagnoles, à St-Domingue et aux Etats-Unis d'Amérique. 2 vols. Paris: Dauvin et Fontaine, $1842-1844$.

Grégoire, Henri. De la noblesse de la peau ou du préjugé des blancs contre la couleur des Africains et celle de leurs descendants noirs et sang-mêlés. Grenoble: Million (1826) 1996, 2002.

Hänni, Eugène. Trois ans chez les Canaques. Odyssée d'un Neuchâtelois autour du monde. Lausanne: Payot, 1908.

Heredia, José María. Niagara y otros textos. Poesia y prosa selectas, edited by Ángel Augier. Caracas: Biblioteca Ayacucho, 1990.

Heredia, José María. Obra poética, edited by Ángel Augier. Havana: Letras Cubanas, 2003.

Heredia, José María. Prosas, edited by Romualdo Santos. Havana: Letras Cubanas, 1980.

Hostos, Eugenio María de. La peregrinación de Bayoán [1863]. Complete works. Vol. 1: Literature, edited by Julio César López. [Rio Piedras]: Editorial de la Universidad de Puerto Rico, 1988.

Hostos, Eugenio María de. "Pages from the Hostos Diary."The Hostos Archives. Bronx, NY: Hostos Community College website. http://commons.hostos.cuny.edu/archives/works-byhostos/pages-from-the-hostos-diary/. Accessed 16 November 2016.

Hostos, Eugenio María de. “¿Cuál de las dos formas de gobierno, monarquía o república, realiza mejor el ideal del derecho?” Obras completas. 20 vols. San Juan: Instituto de Cultura Puertorriqueña, 1969 (Facsimile of edition of La Habana: Cultural S.A., 1939). Vol. 1.

Hostos, Eugenio María de. “Lo que intentó Bolívar.” La Opinión Nacional, 21 December 1876. Hostos, Eugenio María de. Moral social. Sociología. Caracas: Biblioteca Ayacucho, 1982.

Hugo, Victor. Bug-Jargal ou la Révolution haitienne. Les deux versions du roman 1818 et 1826 [1818 and 1826 versions of the novel], edited by Roger Toumson, Fort-de-France: Désormeaux, 1979.

Hugo, Victor. The Letters of Victor Hugo: From Exile, and after the Fall of the Empire, edited by Paul Meurice. Boston: Houghton, Mifflin, 1898. 
Hugo, Victor. "La Mer et le vent." “Cet immense rêve de l’océan ...”. Paysages de mer et autres sujets marins par Victor Hugo (Exhibition catalogue, Maison de Victor Hugo, December 2nd, 2005 to March 5th, 2006), edited by Pierre Georgel. Paris: Les Musées de la Ville de Paris, 2005.

Hugo, Victor. "Preface to Cromwell." Prefaces and Prologues, vol. 39, edited by Charles W. Eliot, The Harvard Classics. New York: P.F. Collier \& Son, 1909-1914; available online at http://www.bartleby.com/39/40.html. Accessed 6 November 2016. ("Préface de Cromwell," cited in Chantal Maignan-Claverie. Le métissage dans la littérature des Antilles françaises. Le complexe d'Ariel. Paris: Karthala, 2005.)

Hugo, Victor. The Toilers of the Sea, translated by Mary Artois. Chicago: W. Irving Way, 1892. (Les travailleurs de la mer [1866]. 3 vols. Paris: Émile Testard, 1891-1892.)

Hurlbert, William Henry (writing as William Henry Hurlbut). "The Exile's Hymn.” (Translation of "Himno del desterrado" by José María Heredia.) Poems of Places: An Anthology in 31 Volumes, edited by Henry Wadsworth Longfellow. Boston: James R. Osgood \& Co., 1876-79; available online at http://www.bartleby.com/270/14/112.html. Accessed 14 November 2016.

Hurlbert, William Henry. "The Poetry of Spanish America." The North American Review 68, 1849, 129-159.

Isaacs, Jorge. María [1867], edited by Donald MacGrady. Madrid: Cátedra, 2007.

Jomard, Edme François. Études géographiques et historiques sur l'Arabie. Paris: Firmin Didot Frères, 1839.

Labat, Jean-Baptiste. Nouveau voyage aux isles de l'Amérique. 6 vols. Paris: Chez Guillaume Cavelier, 1722.

Labat, Jean-Baptiste. Nouveau voyage aux isles de l'Amérique. 8 vols. Paris: Chez J.B. Delespine, 1742.

Lanusse, Armand, editor. Les Cenelles. A Collection of Poems [1845]. Bilingual edition. Translated and with a preface by Regine Latortue and Gleason Rex W. Adams. Boston: Hall, 1979.

Lanusse, Armand. "Besoin d'écrire"/“Compulsion to Write." Les Cenelles. A Collection of Poems [1845], edited by Armand Lanusse. Bilingual edition. Translated and with a preface by Regine Latortue and Gleason Rex W. Adams. Boston: Hall, 1979, 120-123.

Lanusse, Armand. "Introduction." Les Cenelles. A Collection of Poems [1845], edited by Armand Lanusse. Bilingual edition. Translated and with a preface by Regine Latortue and Gleason Rex W. Adams. Boston: Hall, 1979, xxxvi-xli.

Laslo, Pablo, editor. Breve antología de la poesía filipina. Poetas habla española, with a preface by Luis G. Miranda. Mexico City: B. Costa-Amic, 1966.

Le Clézio, Jean-Marie Gustave. L'extase matérielle. Paris: Gallimard, 1967.

Le Clézio, Jean-Marie Gustave. Raga. Paris: Seuil, 2007.

Lerminier, [Eugène]. "Des rapports de la France avec le monde." Revue des deux mondes, initial period, series 4, no. 8, 1836, pp. 326-343.

Lespinasse, Beauvais. "Le Chevalier de Mauduit." Part 1, "30 octobre 1790-Le comité de l'ouest." Revue des Colonies, October 1836, pp. 166-170; Part 2, "Madame Martin et Schelec." Revue des Colonies, November 1836, pp. 206-211; Part 3, “4 mars 1791." Revue des Colonies, December 1836, pp. 245-248.

Levilloux, J. Les créoles ou La vie aux Antilles (1835), followed by Thérèse Bentzon, Yette. Morne-Rouge: Éditions des Horizons Caraïbes, 1977. 
Loti, Pierre. L'île de Pâques. Journal d'un aspirant de la Flore [1899]. Saint-Cyr-sur-Loire: C. Pirot, 2006.

Loti, Pierre. The Marriage of Loti, translated by Clara Bell. London: T. Werner Laurie, 1915. (Le mariage de Loti [1880]. Paris: Flammarion, 1991.)

Louandre, Charles. "De l'association littéraire et scientifique en France." Revue des deux mondes, initial period, series 4, no. 16, 1846, pp. 512-537.

Manifeste pour les "produits” de haute nécessité, Martinique-Guadeloupe-Guyane-Réunion. Signataires: Ernest Breleur, Patrick Chamoiseau, Serge Domi, Gérard Delver, Édouard Glissant, Guillaume Pigeard de Gurbert, Olivier Portecop, Olivier Pulvar, Jean-Claude William. Paris: Éditions Galaade, co-published with l'Institut du Tout-Monde, 2009. Available online at: http://www.tlaxcala.es/detail_artistes.asp?lg=fr\&reference=300. Accessed 23 June 2016.

Manzano, Francisco. Autobiografía del esclavo poeta y otros escritos [1835], edited by William Louis. Frankfurt am Main, Madrid: Vervuert, Iberoamericana, 1997.

Martínez, Melchor. Memoria histórica sobre la Revolución de Chile. Desde el cautiverio de Fernando VII hasta 1814, edited by Guillermo Feliú Cruz. 2 vols. Santiago de Chile: Biblioteca Nacional, 1964.

Maynard de Queilhe, Louis de. Outre-mer. 2 volumes. Paris: Renduel, 1835.

Mazade, Charles de. "La société et la littérature à Cuba." Revue des deux mondes, new period, series 1, vol. XII, 1851, pp. 1017-1035.

Nau, Ignace. Isalina ou Une scène créole [1836]. Port-au-Prince: Choucoune, 2000.

Ortiz, Fernando: Contrapunteo cubano del tabaco y el azúcar. [Habana:] Dir. de publicaciones, Universidad central de Las Villas, 1963.

Picquenard, Jean-Baptiste. Adonis suivi de Zoflora et de documents inédits, edited by Chris Bongie. Paris: L'Harmattan, 2006.

Prévost de Sansac, Auguste. Les amours de Zémédare et Carina et description de l'île de Martinique [1806]. Followed by Louis-Xavier Eyma. Emmanuel, edited by Auguste Joyau. Morne-Rouge: Edition des Horizons Caraïbes, 1977.

Questy, Joanni. "Monsier Paul." La Tribune de la Nouvelle-Orléans, 25 October to 3 November 1867. Available online at http://www.centenary.edu/french/textes/paul.html. Accessed 23 June 2016.

Raynal, Guillaume-Thomas. Histoire philosophique et politique des établissemens et du commerce des européens dans les deux Indes. 10 vols. Geneva, 1781.

Raynal, Guillaume-Thomas. Essai sur l'administration de St. Domingue. [Publisher not identified.] 1785.

Raynal, Guillaume, and Denis Diderot. Die Geschichte beider Indien. Compiled, with illustrations and an afterword, by Hans-Jürgen Lüsebrink. Nördlingen: Greno, 1988.

Recueils de Reglemens, Edits, Declarations et Arretes, Concernant le Commerce, l'Administration de la Justice \& la Police des Colonies Françaises de l'Amérique, \& les Engagés. Avec le Code Noir et l'Addition audit Code. Paris: Chez les Libraires Associez 1745. Available online at: http://www.archive.org/stream/recueilsdereglem00fran\#page/ n3/mode/2up. Accessed 26 June 2016.

Rousseau, Jean-Jacques. Emile: Or, On Education, translated by Allan Bloom. New York: Basic, 1979. (Émile. Éducation. Morale. Botanique. CEuvres complètes, vol. IV, edited by Bernard Gagnebin and Marcel Raymond. Paris: Gallimard, 1999.) 
Rousseau, Jean-Jacques. On the Social Contract, with Geneva Manuscript and Political Economy, edited by Roger D. Masters and Judith R. Masters. New York: St. Martin's Press, 1978. (Du contrat social ou Essai sur la forme de la République. Manuscrit de Genève. Book 1, Chapter 2 [n.d.]. Available online at: http://philo.record.pagesperso-or ange.fr/contrat/geneve.htm. Accessed 10 May 2010.)

Rousseau, Jean-Jacques. The Social Contract, translated by Maurice Cranston.

Harmondsworth, Middlesex, England: Penguin, 1968. (Du contrat social. Écrits

politiques. Euvres complètes. Vol. III, edited by Bernard Gagnebin und Marcel Raymond.

Paris: Gallimard, 1996.)

Rousseau, Jean-Jacques. Émile. Éducation. Morale. Botanique. Complete works, vol. 4, edited by Bernard Gagnebin and Marcel Raymond. Paris: Gallimard, 1999.

Séjour, Victor. "The Mulatto," translated by Philip Barnard. The Norton Anthology of African

American Literature, 2nd edition, edited by Henry Louis Gates, Jr. and Nellie Y. McKay.

New York: Norton, 2004, pp. 78-82. (“Le mulâtre.” Revue des Colonies, March 1837,

pp. 376 -392.)

Tapia y Rivera, Alejandro. La palma del cacique. Leyenda histórica de Puerto Rico y Poesías.

La leyenda de los veinte años. A orillas del Rhin. Mexico: Orion, 1977.

Torabully, Khal. Cale d'étoiles, Coolitude. Sainte-Marie: Azalées, 1992.

Torabully, Khal. Chair corail, fragments coolies. Petit-Bourg (Guadeloupe): Ibis Rouge, 1999.

\section{X.2 Ethnological Journals}

Revue des Colonies, edited by Cyrille Bissette.

Revue des deux mondes.

Revue encyclopédique, ou analyse raisonnée des productions les plus remarquables dans la politique, les sciences, l'industrie et les beaux-arts, edited by Marc-Antoine Jullien.

Paris: Bureau de la Revue Encyclopédique, 1 (1819) -61 (1835).

\section{X.3 Secondary Literature}

Abel, Johanna. “'Aunque la virgen sea blanca, píntame angelitos negros.' Paradoxien in den kolonialen Schönheitsdiskursen der hispano-karibischen Literatur des 19. Jahrhunderts." Grenzgänge. Beiträge zu einer modernen Romanistik, vol. 17, no. 33, 2010 (issue title: Die Maskeraden der Schönheit), pp. 15-32.

Abel, Johanna. "Entre Island Hopping e Islas con Alas. Autoras en el Caribe y sus figuraciones archipiélicas en relatos de viaje del siglo XIX." Worldwide. Archipels de la mondialisation. Archipiélagos de la Globalización, edited by Ottmar Ette and Gesine Müller. Frankfurt am Main, Madrid: Vervuert, Iberoamericana, 2012, pp. 231-249.

Abel, Johanna. "Orientalische Dopplungen in der Karibik: Coolitude als inklusives Kreolitätsmodell und seine dissoziativen Dimensionen." Kreolisierung revisited. Debatten um ein weltweites Kulturkonzept, edited by Gesine Müller and Natascha Ueckmann. Bielefeld: transcript, 2013, pp. 65-81. 
Abel, Johanna. "Tagungsbericht zur Konferenz 'Koloniales Kaleidoskop Karibik. Eine Inselwelt im Fokus kultureller Transferprozesse im 19. Jahrhundert' (09.-11. Juli 2009 in Berlin).” Romanistische Zeitschrift für Literaturgeschichte, 2009, pp. 471-479.

Abel, Johanna. Transatlantisches KörperDenken. Reisende Autorinnen in der spanischen Karibik des 19. Jahrhunderts. Berlin: tranvía (Walter Frey), 2015.

Abel, Johanna. "Viajes corporales al Caribe. Autoras del siglo XIX y sus saberes corporizados sobre las culturas." Relaciones caribeñas. Entrecruzamientos de dos siglos = Relations caribéennes, edited by Liliana Gómez and Gesine Müller. Frankfurt am Main: Lang, 2011, pp. 61-68.

Abel, Johanna, and Gesine Müller. "Korallen: Migration und Transozeanität: Khal Torabully/Indian Diaspora." Handbuch Literatur und Raum, edited by Jörg Dünne and Andreas Mahler. Berlin: De Gruyter, 2015, pp. 505-514.

Abel, Johanna, and Gesine Müller. "Cultural Forms of Representation of 'Coolies': Khal Torabully and His Concept of Coolitude." Bonded Labour. Global and Comparative Perspectives (18th-21st Century), edited by Sabine Damir-Geilsdorf, Ulrike Lindner, et al. Bielefeld: transcript, 2016, pp. 219-228.

Adams, Gerry. "Two Island Peoples in the Same Sea of Struggle." Independent Digital News and Media Limited, 20 December 2001. Available online at http://www.independent.co. uk/voices/commentators/gerry-adams-two-island-peoples-in-the-same-sea-of-struggle5363195.html. Accessed 19 September 2016.

Antoine, Régis. La littérature franco-antillaise. Paris: Éd. Karthala, 1992.

Appadurai, Arjun. Fear of Small Numbers: An Essay on the Geography of Anger. Durham: Duke University Press, 2006.

Arnold, Albert James. "Corsaires, Aventuriers, Flibustier et Pirates. Identité Régionale à la Frontière de l'Empire Espagnol dans la Caraïbe.” In Caleidoscopios coloniales.

Transferencias culturales en el Caribe del siglo XIX = Kaléidoscopes coloniaux. Transferts culturels dans les Caraïbes au XIXe siècle, edited by Ottmar Ette and Gesine Müller. Madrid, Frankfurt am Main: Iberoamericana, Vervuert, 2010, pp. 213-227.

Arzalier, Francis. "Changes in Colonial Ideology in France before 1848. From Slavery to Abolitionism." The Abolitions of Slavery. From Léger Félicité Sonthonax to Victor Schœlcher, 1793, 1794, 1848, edited by Marcel Dorigny. Paris: UNESCO, 2003, pp. 261- 271.

Ashcroft, Bill, Gareth Griffiths, and Helen Tiffin. The Empire Writes Back: Theory and Practice in Post-Colonial Literatures. London: Routledge, 1999.

Asholt, Wolfgang. "Projekt Avantgarde und avantgardistische Selbstkritik." Der Blick vom Wolkenkratzer. Avantgarde-Avantgardekritik-Avantgardeforschung, edited by Wolfgang Asholt and Wolfgang Fähnders. Amsterdam, Atlanta: Rodopi, 2000 (Avantgarde Critical Studies no. 14), pp. 97-120.

Astier Loutfi, Martine. Littérature et colonialisme. Paris: Mouton, 1971.

Bachmann-Medick, Doris. Cultural Turns: New Orientations in the Study of Culture, translated by Adam Blauhut. Boston: Walter De Gruyter, 2016.

Bader, Wolfgang. “Martinique, Guadeloupe, Guyane. Eine periphere Literaturgeschichte.” Französisch heute, no. 17, 1986, pp. 182-201.

Bandau, Anja. "Configuraciones atlánticas y modalidades de la circulación de saberes sobre la rebelión de Saint-Domingue entre 1791 y 1810. El caso de 'Mon Odyssée.”' In Caleidoscopios coloniales. Transferencias culturales en el Caribe del siglo XIX = 
Kaléidoscopes coloniaux. Transferts culturels dans les Caraïbes au XIXe siècle, edited by Ottmar Ette and Gesine Müller. Madrid, Frankfurt am Main: Iberoamericana, Vervuert, 2010, pp. 399-419.

Barbéris, Pierre. Chateaubriand, une réaction au monde moderne. Paris: Librairie Larousse, 1976.

Barnett, Marva A., editor. Victor Hugo on Things That Matter. New Haven, London: Yale University Press, 2010.

Benítez Rojo, Antonio. The Repeating Island: The Caribbean and the Postmodern Perspective, translated by James E. Maraniss, Durham, NC: Duke University Press, 1996. (La isla que se repite. Barcelona: Casiopea, 1998.)

Bénot, Yves. "Haïti et la 'Revue encyclopédique.” Haïti 1804. Lumières et ténèbres. Impact et résonances d'une révolution, edited by Leon-François Hoffmann, Frauke Gewecke, et al. Madrid: Iberoamericana, 2008, pp. 99-112.

Berg, Hubert van den, and Walther Fähnders. "Avantgarde als Netzwerk und Projekt.” Metzler Lexikon Avantgarde, edited by Hubert van den Berg and Walther Fähnders. Stuttgart: Metzler, 2009, pp. 11-12.

Bernabé, Jean, Patrick Chamoiseau, and Raphaël Confiant. Éloge de la Créolité [1989]. Paris: Gallimard, 2002.

Blum, Hester, editor. Turns of Event: American Literary Studies in Motion. Philadelphia: University of Pennsylvania Press, 2016.

Blumenberg, Hans. Theorie der Lebenswelt, edited by Manfred Sommer, Berlin: Suhrkamp, 2010.

Blümig, Gabriele. Retour au paysage natal. Zur Natur im postkolonialen Roman der frankophonen Antillen. Diss. Würzburg, 2004. Available online at: https://opus.bib liothek.uni-wuerzburg.de/frontdoor/index/index/docld/1500. Accessed 23 June 2016.

Bonafoux, Luis. Betances. San Juan de P.R.: Instituto de Cultura Puertoriqueña, 1970.

Bongie, Chris. “'C'est du papier ou de l'Histoire en Marche?' The Revolutionary Compromises of a Martiniquan Homme de Couleur, Cyrille-Charles-August Bissette.” Nineteenth Century Contexts no. 23, 2002, pp. 439-473.

Bongie, Chris. Friends and Enemies. The Scribal Politics of Post/Colonial Literature. Liverpool: Liverpool University Press, 2008.

Bongie, Chris. Islands and Exiles. The Creole Identities of Post/Colonial Literature. Stanford: Stanford University Press, 1998.

Bongie, Chris. "Politique, Mémoire, Littérature. L'Universalité fractionniste' d'Haïti au XIXe siècle." In Caleidoscopios coloniales. Transferencias culturales en el Caribe del siglo XIX = Kaléidoscopes coloniaux. Transferts culturels dans les Caraïbes au XIXe siècle, edited by Ottmar Ette and Gesine Müller. Madrid, Frankfurt am Main: Iberoamericana, Vervuert, 2010, pp. 231-252.

Boren, Mark E. Sugar, Slavery, Christianity and the Making of Race. Pompano Beach, FL: Caribbean Studies Press, 2013.

Borrego Plá, María del Carmen. "La influencia de la Francia revolucionaria en México. El texto constitucional de Apatzingán.” América Latina ante la Revolución Francesa, edited by María del Carmen Borrego Plá and Leopoldo Zea. México, D.F.: Univ. Nacional Autónoma de México, 1993, pp. 9-30.

Bragard, Véronique. Transoceanic Dialogues: Coolitude in Caribbean and Indian Ocean Literatures. Frankfurt am Main: Peter Lang, 2008. 
Bremer, Thomas. "Haiti als Paradigma. Karibische Sklavenemanzipation und europäische Literatur." Karibik. Wirtschaft, Gesellschaft und Geschichte, edited by Hanns-Albert Steger and Jürgen Schneider. Munich: Fink, 1982, pp. 319-340.

Bremer, Thomas. "Juan Francisco Manzano y su Autobiografía de un esclavo (Cuba, 1835/1840). La repercusión en Europa." Caleidoscopios coloniales. Transferencias culturales en el Caribe del siglo XIX = Kaléidoscopes coloniaux. Transferts culturels dans les Caraïbes au XIXe siècle, edited by Ottmar Ette and Gesine Müller. Madrid, Frankfurt am Main: Iberoamericana, Vervuert, 2010, pp. 439-448.

Breña, Roberto. El primer liberalismo español y los procesos de emancipación de América, 1808-1824. Una revisión historiográfica del liberalismo hispánico. México, D.F.: El Colegio de México, 2006.

Brickhouse, Anna. Transamerican Literary Relations and the Nineteenth-Century Public Sphere. Cambridge: Cambridge University Press, 2004.

Brutus, Edner. Instruction publique en Haïti: 1492-1945. Port-au-Prince: Imprimerie de l'État, 1948.

Candlin, Kit, and Cassandra Pybus. Enterprising Women: Gender, Race, and Power in the Revolutionary Atlantic. Athens, Georgia: University of Georgia Press (Race in the Atlantic World, 1700-1900), 2014.

Carter, Marina, and Khal Torabully. Coolitude. An Anthology of the Indian Labour Diaspora. London: Anthem Press, 2002.

Castells, Irene. La ciudadanía revolucionaria, 2002. Available online at: http://www.casa taule.org/cast/docs/la_ciudadania_revolucionaria.doc. Accessed 10 May 2010.

Castro Varela, María do Mar, and Nikita Dhawan. Postkoloniale Theorie. Eine kritische Einführung. Bielefeld: transcript, 2005.

Chambers, Stephen M. No God But Gain: The Untold Story of Cuban Slavery, the Monroe Doctrine, and the Making of the United States. London, New York: Verso, 2015.

Chamoiseau, Patrick, and Raphaël Confiant. Lettres créoles. Tracées antillaises et continentales de la littérature: Haïti, Guadeloupe, Martinique, Guyane; 1635-1975. Paris: Hatier, 1991.

Chamoiseau, Patrick, and Raphaël Confiant. "En guise d'introduction: 'Points de vue sur l'évolution de la littérature antillaise.' Entretien avec les écrivains martiniquais Patrick Chamoiseau et Raphaël Confiant (mené par Ottmar Ette et Ralph Ludwig)." Les littératures antillaises-une mosaïque culturelle, edited by Ottmar Ette and Ralph Ludwig. Dossier in Lendemains, no. 67, 1992.

Champion, Jean-Marcel. "30 Floréal Year X. The Restoration of Slavery by Bonaparte." The Abolitions of Slavery. From Léger Félicité Sonthonax to Victor Schœlcher, 1793, 1794, 1848, edited by Marcel Dorigny. Paris: UNESCO, 2003, pp. 229-236.

Chappey, Jean-Luc. La Société des Observateurs de l'Homme (1799-1804) des anthropologues au temps de Bonaparte. Paris: Société des Études Robespierristes, 2002.

Chinea, Jorge L. Raza y trabajo en el Caribe hispánico: los inmigrantes de las Indias occidentales en Puerto Rico durante el ciclo agro-exportador, 1800-1850. Sevilla: Asociación Cultural la otra Andalucía, 2014.

Corzani, Jack. La littérature des Antilles-Guyane Françaises. 6 volumes. Fort-de-France: Desormeaux, 1987. 
Daston, Lorraine. Things that Talk: Object Lessons from Art and Science. New York: Zone Books, 2004.

Deleuze, Gilles, and Félix Guattari. Rhizome: Introduction. Paris: Minuit, 1977.

Delle, James A. The Colonial Caribbean: Landscapes of Power in the Plantation System. New York: Cambridge University Press, 2014.

Démier, Francis. "Slavery, Colonial Economy and French Development Choices during the First Industrialization (1802-1840)." The Abolitions of Slavery. From Léger Félicité Sonthonax to Victor Schœlcher, 1793, 1794, 1848, edited by Marcel Dorigny. Paris: UNESCO, 2003, pp. 237-247.

Desdunes, Rodolphe Lucien. Our People and Our History; A Tribute to the Creole People of Color in Memory of the Great Men They Have Given Us and of the Good Works They Have Accomplished. Baton Rouge: Louisiana State University Press, 1973. (Nos hommes et notre histoire. Notices biographiques accompagnées de réflexions et de souvenirs personnels, hommage à la population créole, en souvenir des grands hommes qu'elle a produits et des bonnes choses qu'elle a accomplies. Montreal: Arbour \& Dupont, 1911.)

Díaz, Roberto Ignacio. “Merlin's Foreign House. The Genres of La Havane.” Cuban Studies, no. 24, 1994, pp. 57-82.

Downey, Arthur T. The Creole Affair: The Slave Rebellion that Led the U.S. and Great Britain to the Brink of War. Lanham: Rowman \& Littlefield, 2014.

Drescher, Seymour. "British Way, French Way. Opinion Building and Revolution in the Second French Slave Emancipation." From Slavery to Freedom. Comparative Studies in the Rise and Fall of Atlantic Slavery, edited by Seymour Drescher, Basingstoke: Macmillan, 1999, pp. $158-195$.

Drescher, Seymour. "Two Variants of Anti-Slavery. Religious Organization and Social Mobilization in Britain and France, 1780-1870." From Slavery to Freedom. Comparative Studies in the Rise and Fall of Atlantic Slavery, edited by Seymour Drescher. Basingstoke: Macmillan, 1999, pp. 35-56.

Duchet, Michèle. Anthropologie et histoire au siècle des lumières. Buffon, Voltaire, Rousseau, Helvétius, Diderot. Paris: Maspero, 1971.

Duchet, Michèle. Le partage des savoirs. Discours historique et discours ethnologique. Paris: Éditions la Découverte, 1985.

Duke Bryant, Kelly. "Black but not African. Francophone Black Diaspora and the Revue des Colonies, 1834-1842." International Journal of African Historical Studies, vol. 40, no. 2, 2007, pp. 251-282.

Duplantier, Jean-Marc Allard. "Nos frères d'outre-golf." Spiritualism, Vodou and the Mimetic Literatures of Haiti and Louisiana. Diss. Louisiana State University 2006. Available online at http://etd.lsu.edu/docs/available/etd-11152006-152550/unrestricted/Du plantier_dis.pdf. Accessed June 232016.

Ecker, Gisela, and Susanne Scholz, editors. Umordnungen der Dinge. Königstein (Taunus): Helmer, 2000.

Enwezor, Okwui. “The Black Box.” Documenta 11 Platform 5: Exhibition. Kassel, 2002, pp. 42 -55 .

Ette, Ottmar. Alexander von Humboldt und die Globalisierung. Frankfurt am Main: Suhrkamp 2009. 
Ette, Ottmar. "Cirilo Villaverde: Cecilia Valdés o La Loma del Angel." Der hispanoamerikanische Roman, edited by Volker Roloff and Harald Wentzlaff-Eggebert. Darmstadt: Wissenschaftliche Buchgesellschaft, 1992, vol. 1, pp. 30-43; $313 \mathrm{f}$.

Ette, Ottmar. "Crossing the Mangrove." Literature on the Move, translated by Katharina Vester. Amsterdam: Rodopi, 2003, pp. 255-294. (“Die Durchquerung der Mangroven." Literatur in Bewegung. Raum und Dynamik grenzüberschreitenden Schreibens in Europa und Amerika. Weilerswist: Velbrück Wissenschaft, 2001, pp. 461-538.)

Ette, Ottmar. "Diskurse der Tropen-Tropen der Diskurse: Transarealer Raum und literarische Bewegungen zwischen den Wendekreisen." Raum und Bewegung in der Literatur. Die Literaturwissenschaften und der Spatial Turn, edited by Wolfgang Hallet and Birgit Neumann. Bielefeld: transcript, 2009, pp. 139-165.

Ette, Ottmar. La escritura de la memoria. Reinaldo Arenas: Textos, estudios y documentación. Frankfurt am Main: Vervuert, 1992.

Ette, Ottmar. "Europäische Literatur(en) im globalen Kontext. Literaturen für Europa." Wider den Kulturenzwang. Migration, Kulturalisierung und Weltliteratur, edited by Özkan Ezli, Dorothee Kimmich, et al. Bielefeld: transcript, 2009, pp. 257-296.

Ette, Ottmar. "Figuren und Funktionen des Lesens in Guillaume-Thomas Raynals Histoire des deux Indes." Ex nobili philologorum officio. Festschrift für Heinrich Bihler zu seinem 80. Geburtstag, edited by Dietrich Briesemeister and Axel Schönberger. Berlin: Domus Ed. Europaea, 1998, pp. 589-610.

Ette, Ottmar. Konvivenz. Literatur und Leben nach dem Paradies. Berlin: Kulturverlag Kadmos, 2012.

Ette, Ottmar. Literature on the Move, translated by Katharina Vester. Amsterdam: Rodopi, 2003. (Literatur in Bewegung. Raum und Dynamik grenzüberschreitenden Schreibens in Europa und Amerika. Weilerswist: Velbrück Wissenschaft, 2001.)

Ette, Ottmar. "Eine Literatur ohne festen Wohnsitz. Fiktionen und Friktionen der kubanischen Literatur im 20. Jahrhundert." Romanistische Zeitschrift für Literaturgeschichte / Cahiers d'Histoire des Littératures Romanes (Heidelberg), vol. 28, nos. 3-4, 2004, pp. 457-481.

Ette, Ottmar. "Literaturwissenschaft als Lebenswissenschaft." Lendemains, no. 125, 2007, pp. $7-32$.

Ette, Ottmar. "Le monde transarchipélien de la Caraïbe coloniale." Caleidoscopios coloniales. Transferencias culturales en el Caribe del siglo XIX = Kaléidoscopes coloniaux. Transferts culturels dans les Caraïbes au XIXe siècle, edited by Ottmar Ette and Gesine Müller. Madrid, Frankfurt am Main: Iberoamericana, Vervuert, 2010, pp. 23-64.

Ette, Ottmar. "Réflexions européennes sur deux phases de mondialisation accélérée chez Cornelius de Pauw, Georg Forster, Guillaume-Thomas Raynal et Alexandre de Humboldt." HiN. Alexander von Humboldt im Netz. Internationale Zeitschrift für Humboldt-Studien (Potsdam - Berlin), vol. 11, no. 21, 2010, pp.1-28. Available online at: http://www.hinonline.de/index.php/hin/article/view/143. Accessed 17 July 2016.

Ette, Ottmar. “'Traición, naturalmente.' Espacio literario, poetología implícita en 'La Loma del Angel,' de Reinaldo Arenas.” Reinaldo Arenas. Recuerdo y Presencia, edited by Reinaldo Sánchez. Miami: Ediciones Universal, 1994, pp. 87-107.

Ette, Ottmar. TransArea. Eine literarische Globalisierungsgeschichte. Berlin, Boston: De Gruyter, 2012.

Ette, Ottmar. "Von Inseln, Grenzen und Vektoren. Versuch über die fraktale Inselwelt der Karibik." Grenzen der Macht-Macht der Grenzen. Lateinamerika im globalen Kontext, 
edited by Marianne Braig, Ottmar Ette, et al. Frankfurt am Main: Vervuert, 2005, pp. 135-180.

Ette, Ottmar. Weltbewusstsein. Alexander von Humboldt und das unvollendete Projekt einer anderen Moderne. Göttingen: Velbrück Wissenschaft, 2002.

Ette, Ottmar. Writing-between-Worlds: Transarea Studies and the Literatures-without-a-Fixed-Abode. Translated by Vera M. Kutzinski. Boston: De Gruyter, 2016. (ZwischenWeltenSchreiben. Literaturen ohne festen Wohnsitz. Berlin: Kulturverlag Kadmos, 2005.)

Ette, Ottmar. ZusammenLebensWissen. List, Last und Lust literarischer Konvivenz im globalen Maßstab. Berlin: Kadmos, 2010.

Ette, Ottmar, and Werner Mackenbach, et al., editors. Trans(it)Areas. Convivencias en Centroamérica y el Caribe. Un simposio transareal. Berlin: tranvía (Walter Frey), 2011.

Ette, Ottmar, and Gesine Müller, editors. New Orleans and the Global South. Caribbean, Creolization, Carnival. Hildesheim: Olms, 2017.

Fabre, Michel. From Harlem to Paris. Black American Writers in France, 1840-1980. Urbana: University of Illinois Press, 1991.

Fabre, Michel. "The New Orleans Press and French-Language Literatures by Creoles of Color." Multilingual America. Transnationalism, Ethnicity, and the Languages of American Literature, edited by Werner Sollers. New York: New York University Press, 1998, pp. $29-49$.

Faessel, Sonia. Visions des îles: Tahiti et l'imaginaire européen. Du mythe à son exploitation littéraire (XVIII $-X X I^{e}$ siècles). Paris: L'Harmattan, 2006.

Fink-Eitel, Hinrich. Die Philosophie und die Wilden. Über die Bedeutung des Fremden für die europäische Geistesgeschichte. Hamburg: Junius, 1994.

Fischer, Sibylle. Modernity Disavowed. Haiti and the Cultures of Slavery in the Age of Revolution. Durham, NC: Duke University Press, 2004.

Fleischmann, Ulrich. Ideologie und Wirklichkeit in der Literatur Haitis. Berlin: Colloquium-Verlag, 1969.

Flory, Céline. De l'esclavage à la liberté forcée: histoire des travailleurs africains engagés dans la Carä̈be française au XIXe siècle. Paris: Éditions Karthala, 2015.

Foucault, Michel. The Order of Things: An Archaeology of the Human Sciences. New York: Vintage, 1994.

Fradera, Josep. Colonias para después de un Imperio. Barcelona: Ed. Bellaterra, 2005.

Garber, Jörn, and Heinz Thoma. Zwischen Empirisierung und Konstruktionsleistung.

Anthropologie im 18. Jahrhundert. Tübingen: Niemeyer, 2004.

Gazmuri Riveros, Cristián. "Libros e ideas políticas francesas en la gestación de la independencia de Chile." América Latina ante la Revolución Francesa, edited by María del Carmen Borrego Plá and Leopoldo Zea. México, D.F.: Univ. Nacional Autónoma de México, 1993, pp. 81-108.

Gaztambide Géigel, Antonio. "La geopolítica del antillanismo en el Caribe del siglo XIX. Memorias. Revista Digital de Historia y Arqueología desde el Caribe 4, 8, 2007, 41-74. Available online at: http://www.redalyc.org/articulo.oa?id=85540808. Accessed 23 June 2016.

Gewecke, Frauke. "Les Antilles face à la Révolution haïtienne. Césaire, Glissant, Maximin.” Haïti 1804. Lumières et ténèbres. Impact et résonances d'une révolution, edited by 
Leon-François Hoffmann, Frauke Gewecke, et al. Madrid: Iberoamericana, 2008, pp. 251-266.

Gewecke, Frauke. Die Karibik. Zur Geschichte, Politik und Kultur einer Region. Frankfurt am Main: Vervuert, 2007.

Gewecke, Frauke. "Saint-Domingue/Haití-Santo Domingo. Proyectos de una isla/nación une et indivisible." Caleidoscopios coloniales. Transferencias culturales en el Caribe del siglo $X I X=$ Kaléidoscopes coloniaux. Transferts culturels dans les Caraïbes au XIXe siècle, edited by Ottmar Ette and Gesine Müller. Madrid, Frankfurt am Main: Iberoamericana, Vervuert, 2010, pp. 253-281.

Gewecke, Frauke. “Der Titel als Chiffre einer Subversion. 'Moi, Tituba, sorcière ... Noire de Salem' von Maryse Condé.” Titel-Text-Kontext. Randbezirke des Textes. Festschrift für Arnold Rothe zum 65. Geburtstag, edited by Jochen Mecke and Arnold Rothe. Glienicke, Berlin: Galda + Wilch, 2000, pp. 159-177.

Gewecke, Frauke. Der Wille zur Nation. Nationsbildung und Entwürfe nationaler Identität in der Dominikanischen Republik. Frankfurt am Main: Vervuert, 1996.

Gilroy, Paul. After Empire. Melancholia or Convivial Culture? London: Routledge, 2004.

Gilroy, Paul. Postcolonial Melancolia. New York: Columbia Univ. Press, 2005.

Gilroy, Paul. The Black Atlantic. Modernity and Double Consciousness. Cambridge: Harvard University Press, 1993.

Gisler, Antoine. L'esclavage aux Antilles françaises XVIIe-XIXe siècle. Revised and corrected edition, reprint of 1965 Fribourg, Switzerland edition. Paris: Karthala, 1981.

Glissant, Édouard. Caribbean Discourse: Selected Essays. Translated and with an introduction by J. Michael Dash. Charlottesville: University Press of Virginia, 1989.

Glissant, Édouard. La cohée du Lamentin. Poétique. V. Paris: Gallimard, 2005.

Glissant, Édouard. Le discours antillais. Paris: Gallimard, 1997.

Glissant, Édouard. Introduction à une poétique du divers. Paris: Gallimard, 1996.

Glissant, Édouard. Kultur und Identität. Ansätze zu einer Poetik der Vielheit. Translated by Beate Thill. Heidelberg: Wunderhorn, 2005.

Glissant, Édouard. Mémoires des esclavages. La fondation d'un centre national pour la mémoire des esclavages et de leurs abolitions. Paris: Gallimard, 2007.

Glissant, Édouard. Poetics of Relation, translated by Betsy Wing. Ann Arbor: University of Michigan, 1997. (Poétique de la relation. Paris: Gallimard, 1990.)

Glissant, Édouard. Tout-monde. Paris: Gallimard, 2003.

Glissant, Édouard, and Patrick Chamoiseau. "De loin.” Potomitan. Site de promotion des cultures et des langues créoles. http://www.potomitan.info/articles/deloin.php. Accessed June 232016.

Graham, Maryemma, and Wilfried Raussert. Mobile and Entangled America(s). Burlington, VT: Ashbate Publishing Company, 2016.

Haddox, Thomas F. “The 'Nous' of Southern Catholic Quadroons. Racial, Ethnic, and Religious Identity in Les Cenelles." American Literature, vol. 73, no. 4, 2001, pp. 757-778.

Haesendonck, Kristian von, and Theo D'Haen. Caribbeing. Comparing Caribbean Literatures and Cultures. Amsterdam: Rodopi, 2014.

Hallward, Peter. Absolutely Postcolonial. Writing Between the Singular and the Specific. Manchester: Manchester University Press, 2001. 
Hartog, François. Regimes of Historicity: Presentism and Experiences of Time, translated by Saskia Brown. New York: Columbia University Press, 2015. (Régimes d'historicité. Présentisme et expériences du temps. Paris: Seuil, 2003.)

Hassauer, Friederike. Santiago. Schrift, Körper, Raum, Reise. Eine medienhistorische Rekonstruktion. Munich: Fink, 1993.

Hau'Ofa, Epeli. We Are the Ocean. Selected Works. Honolulu: University of Hawaii Press, 2008.

Hernández Sánchez-Barba, Mario. "Las cortes españolas ante la abolición de la esclavitud en las Antillas. Opinión institucional ante un tema de política social." Quinto Centenario, no. 8,1985 , pp. $15-36$.

Hoffmann, Léon-François. Littérature d'Haïti. Vanves: Edicef, 1995.

Hoffmann, Léon-François. Le Nègre romantique. Personnage littéraire et obsession collective. Paris: Payot, 1973.

Hölz, Karl. Zigeuner, Wilde und Exoten. Fremdbilder in der französischen Literatur des 19. Jahrhunderts. Berlin: Schmidt, 2002.

lanes, Raúl. "La esfericidad del papel. Gertrudis Gómez de Avellaneda, la condesa de Merlín y la literatura de viajes." Revista Iberoamericana, vol. LXIII, no. 178-179, January to June 1997, pp. 209-218.

James, C.L.R. The Black Jacobins: Toussaint L'Ouverture and the San Domingo Revolution. London: Penguin, (1938) 2001.

Johnson, Jerah. “Les Cenelles. What's in a Name?" Louisiana History, vol. 31, no. 4, 1990, pp. 407-410.

Johnson, Sara Elizabeth. The Fear of French Negroes: Transcolonial Collaboration in the Revolutionary Americas. Berkeley: University of California Press, 2012.

Kapur, Geeta. When Was Modernism: Essays on Contemporary Cultural Practice in India. New Dehli: Tulika, 2000.

Kielstra, Paul Michael. The Politics of Slave Trade Suppression in Britain and France, 1814-48. Diplomacy, Morality and Economics. Basingstoke, Hampshire: Macmillan, 2000.

Kirsch, Fritz Peter. Epochen des französischen Romans. Vienna: WUV, 2000.

Klein, Martin A. Historical Dictionary of Slavery and Abolition. Lanham: Scarecrow Press, 2002.

König, Torsten. “Édouard Glissants pensée archipélique. Zwischen Metapher und poetischem Prinzip." Raum-Bewegung-Passage. Postkoloniale frankophone Literaturen, edited by Gesine Müller and Susanne Stemmler. Tübingen: Narr, 2009, pp. 113-130.

König, Torsten. "L'imaginaire géopolitique de la Polynésie dans la littérature française: de Bougainville à Chantal T. Spitz.” Worldwide. Archipels de la mondialisation.

Archipiélagos de la globalización, edited by Ottmar Ette and Gesine Müller. Madrid, Frankfurt am Main: Iberoamericana, Vervuert, 2012, pp. 129-148.

Korte, Barbara. Der englische Reisebericht. Von der Pilgerfahrt bis zur Postmoderne. Darmstadt: Wissenschaftliche Buchgesellschaft, 1996.

Küpper, Joachim. Ästhetik der Wirklichkeitsdarstellung und Evolution des Romans von der französischen Spätaufklärung bis zu Robbe-Grillet. Ausgewählte Probleme zum Verhältnis von Poetologie und literarischer Praxis. Stuttgart: Steiner, 1987.

Latour, Bruno. Politics of Nature: How to Bring the Sciences into Democracy, translated by Catherine Porter. Cambridge: Harvard University Press, 2009. 
Lehnert, Gertrud. "Des 'robes à la turque' et autres orientalismes à la mode." Paris Croisé, ou comment le monde extra-européen est venu dans la capitale française (1760-1800), edited by Anja Bandau, Marcel Dorigny, et al. Paris: Karthala, 2010, pp. 183-200.

Leonard, Adrian, and David Pretel, editors. The Caribbean and the Atlantic World Economy: Circuits of Trade, Money and Knowledge, 1650-1914. New York: Palgrave Macmillan (Cambridge Imperial and Post-Colonial Studies Series), 2015.

Lepenies, Wolf. Das Ende der Naturgeschichte. Wandel kultureller Selbstverständlichkeiten in den Wissenschaften des 18. und 19. Jahrhunderts. Munich: Hanser, 1976.

Llorens, Irma. Nacionalismo y literatura. Constitución e institucionalización de la "República de las Letras Cubanas." Lérida: Asociación Española de Estudios Literarios Hispanoamericanos, 1998.

Lowe, John Wharton. Calypso Magnolia: The Crosscurrents of Caribbean and Southern Literature. Chapel Hill: The University of North Carolina Press, 2016.

Ludwig, Ralph. Frankokaribische Literatur. Eine Einführung. Tübingen, 2008.

Ludwig, Ralph, and Dorothee Röseberg. "Einleitende Fragen." Tout-Monde: Interkulturalität, Hybridisierung, Kreolisierung. Kommunikations- und gesellschaftstheoretische Modelle zwischen "alten" und "neuen" Räumen, edited by Ralph Ludwig and Dorothee Röseberg. Frankfurt am Main: Lang, 2010, pp. 7-30.

Lüsebrink, Hans-Jürgen. "Aufklärerisches Erkenntnispotential versus institutionelle Erkenntnisschranken. Zur Geschichtsschreibung Henri Grégoires (1751-1831).” Von der Aufklärung zum Historismus. Zum Strukturwandel des historischen Denkens, edited by Horst-Walter Blanke and Jörn Rüsen. Paderborn: Schöningh, 1985, pp. 203-218.

Lüsebrink, Hans-Jürgen. Das Europa der Aufklärung und die außereuropäische koloniale Welt. Göttingen: Wallstein, 2006.

Lüsebrink, Hans-Jürgen. Interkulturelle Kommunikation. Interaktion, Fremdwahrnehmung, Kulturtransfer. Stuttgart: Metzler, 2008.

Lüsebrink, Hans-Jürgen. "Missionarische Fremdheitserfahrung und anthropologischer Diskurs. Zu den Nachrichten von der Amerikanischen Halbinsel Californien (1772) des elsässischen Jesuitenmissionars Johann Jakob Baegert." Lateinamerika. Orte und Ordnungen des Wissens. Festschrift für Birgit Scharlau, edited by Sabine Hofmann and Monika Wehrheim. Tübingen: Gunter Narr Verlag, 2004, pp. 69-82.

Lüsebrink, Hans-Jürgen. “'Negrophilie’ und Paternalismus. Die Beziehungen Henri Grégoires zu Haiti (1790-1831)." Der karibische Raum zwischen Selbst- und Fremdbestimmung. Zur karibischen Literatur, Kultur und Gesellschaft, edited by Reinhard Sander. Frankfurt am Main: Peter Lang, 1984, pp. 99-108.

Lüsebrink, Hans-Jürgen. "Transfers culturels et légitimation postcoloniale du pouvoir. L'émergence de la presse et de la littérature haïtienne pendant le règne du Roi Christophe en Haïti." Caleidoscopios coloniales. Transferencias culturales en el Caribe del siglo XIX = Kaléidoscopes coloniaux. Transferts culturels dans les Caraïbes au XIXe siècle, edited by Ottmar Ette and Gesine Müller. Madrid, Frankfurt am Main: Iberoamericana, Vervuert, 2010, pp. 305-325.

Magerski, Christine. Theorien Der Avantgarde: Gehlen-Bürger-Bourdieu-Luhmann. Wiesbaden: VS Verlag für Sozialwissenschaften, 2011.

Maignan-Claverie, Chantal. Le métissage dans la littérature des Antilles françaises. Le complexe d'Ariel. Paris: Karthala, 2005. 
Malena, Anne. The Negotiated Self. The Dynamics of Identity in Francophone Caribbean Narrative. New York: Lang, 1999.

Maluquer de Motes, Jordi. "Abolicionismo y resistencia a la abolición en la España del siglo XIX.” Anuario de Estudios Americanos, no. 53, 1986, pp. 311-331.

Martínez-San Miguel, Yolanda, Ben Sifuentes-Jáuregui, and Marisa Belausteguigoitia, editors. Critical Terms in Caribbean and Latin American Thought: Historical and Institutional Trajectories. Houndmills, Basingstoke, Hampshire; New York, NY: Palgrave Macmillan, 2016.

Mateos, Ana. "Dialéctica para una voz propia en Cecilia Valdés." Caleidoscopios coloniales. Transferencias culturales en el Caribe del siglo XIX = Kaléidoscopes coloniaux. Transferts culturels dans les Caraïbes au XIXe siècle, edited by Ottmar Ette and Gesine Müller. Madrid, Frankfurt am Main: Iberoamericana, Vervuert, 2010, pp. 103-120.

Matzat, Wolfgang. Diskursgeschichte der Leidenschaft. Zur Affektmodellierung im französischen Roman von Rousseau bis Balzac. Tübingen: Narr, 1990.

McIntosh, Malachi. Emigration and Caribbean Literature. New York: Palgrave Macmillan, 2015. Méndez Rodenas, Adriana. Gender and Nationalism in Colonial Cuba. The Travels of Santa Cruz y Montalvo, Condesa de Merlin. Nashville: Vanderbilt University Press, 1998.

Méndez Rodenas, Adriana. "A Journey to the (Literary) Source. The Invention of Origins in Merlin's Viaje a La Habana.” New Literary History, vol. 21, no. 3, 1990, pp. 707-31.

Méndez Rodenas, Adriana. "Voyage to La Havane. The Countess of Merlín's Preview of National Identity." Cuban Studies, no. 16, 1986, pp. 71-99.

Mesnard, Éric. "Resistance Movements in the French Colonies. The Bissette Affair (1823-1827)." The Abolitions of Slavery. From Léger Félicité Sonthonax to Victor Schœlcher, 1793, 1794, 1848, edited by Marcel Dorigny. Paris: UNESCO, 2003, pp. $255-260$.

Meyer-Krentler, Leonie. "El Bois-Caïman y la mitificación de la figura negra en Les Créoles ou la Vie aux Antilles de J. Levilloux." Relaciones caribeñas. Entrecruzamientos de dos siglos = Relations caribéennes, edited by Liliana Gómez and Gesine Müller. Frankfurt am Main: Lang, 2011, pp. 69-88.

Meyer-Krentler, Leonie. Die Idee des Menschen in der Karibik. Mensch und Tier in französisch- und spanischsprachigen Erzähltexten des 19. Jahrhunderts. Berlin: tranvía (Walter Frey), 2013.

Meyer-Krentler, Leonie. "Los perros ingleses y los perros esclavos. Exclusión, animalización y convivencia en Cecila Valdés de Cirilo Villaverde." Worldwide. Archipels de la mondialisation. Archipiélagos de la globalización, edited by Ottmar Ette and Gesine Müller. Frankfurt am Main, Madrid: Vervuert, Iberoamericana, 2012, pp. 194-210.

Middelanis, Carl Hermann: Imperiale Gegenwelten. Haiti in den französischen Text- und Bildmedien 1848-1870. Frankfurt am Main: Vervuert, 1996.

Mignolo, Walter D. Local Histories/Global Designs. Coloniality, Subaltern Knowledges, and Border Thinking. Princeton: Princeton University Press, 2012.

Mignolo, Walter D. "La razón postcolonial. Herencias coloniales y teorías postcoloniales." Postmodernidad y postcolonialidad. Breves reflexiones sobre Latinoamérica, edited by Alfonso de Toro. Madrid: Vervuert, Iberoamericana, 1997, pp. 51-70.

Misevich, Philip, and Kristin Mann. The Rise and Demise of Slavery and the Slave Trade in the Atlantic World. Rochester, NY: University of Rochester Press, 2016. 
Möllers, Nina. Kreolische Identität. Eine amerikanische "Rassengeschichte" zwischen Schwarz und Weiß. Die Free People of Color in New Orleans. Bielefeld: transcript, 2008.

Molloy, Sylvia. At Face Value. Autobiographical Writing in Spanish America. Cambridge: Cambridge University Press, 1991.

Mouffe, Chantal. On the Political. London: Routledge, 2005.

Müller, Gesine. Die Boom-Autoren heute. García Márquez, Fuentes, Vargas Llosa, Donoso und ihr Abschied von den "großen identitätsstiftenden Entwürfen." Frankfurt am Main: Vervuert, 2004.

Müller, Gesine. "El Caribe como caleidoscopio de dinámicas coloniales (1789-1886)." Relaciones caribeñas. Entrecruzamientos de dos siglos = Relations caribéennes, edited by Liliana Gómez and Gesine Müller. Frankfurt am Main: Lang, 2011, pp. 13-36.

Müller, Gesine. "Chateaubriand und Hugo in der Karibik. Literarische Inszenierungen des Anderen oder die omnipräsente Rezeption der französischen Romantik." Das Andere Schreiben. Diskursivierungen von Alterität in Texten der Romania (16.-19. Jahrhundert), edited by Susanne Greilich and Karen Struve. Würzburg: Königshausen \& Neumann, 2013, pp. 213-224.

Müller, Gesine. "Chorégraphies des paysages coloniaux: Victor Hugo et Pierre Loti." Chorégraphies du paysage littéraire, edited by Ottmar Ette and Gesine Müller. Dossier in Lendemains, vol. 145, no. 37, 2012, pp. 88-101.

Müller, Gesine. "Entre la francofilia y las aspiraciones de autonomía: Una mirada desde el Caribe sobre las diferentes constelaciones postcoloniales." Escribiendo la Independencia. Perspectivas postcoloniales sobre la literatura hispanoamericana del siglo XIX, edited by Robert Folger and Stephan Leopold. Frankfurt am Main, Madrid: Vervuert, Iberoamericana, 2010, pp. 125-139.

Müller, Gesine. “J.-M.G. Le Clézio, Edouard Glissant, Epeli Hau’Ofa: Avantgarden in Ozeanien." Avantgarde und Modernismus. Dezentrierung, Subversion und Transformation im literarisch-künstlerischen Feld, edited by Wolfgang Asholt. Berlin: De Gruyter, 2014, pp. 169-180.

Müller, Gesine. "Koloniale Achsen und ihre literarischen Repräsentationen in der Karibik im 19. Jahrhundert: Utopien vom Zusammenleben in transkolonialen Dimensionen." Literarische Stadtutopien zwischen totalitärer Gewalt und Ästhetisierung, edited by Barbara Ventarola. München: Meidenbauer, 2011, pp. 321-338.

Müller, Gesine. "Konvivenz und Relationalität im französischen Kolonialreich. Atlantische und pazifische Meerlandschaften im Vergleich." Brücken bauen - Kulturwissenschaft aus interkultureller und multidisziplinärer Perspektive. Festschrift für Dorothee Röseberg zum 65. Geburtstag, edited by Marie-Therese Mäder, Chantal Metzger, et al. Bielefeld: transcript, 2016, pp. 257-272.

Müller, Gesine. "La littérature jaune. Gustave d’Alaux und frühe Zeugnisse haitianischer Literaturgeschichtsschreibung." Wort - Macht - Stamm, edited by Markus Messling and Ottmar Ette. München: Fink, 2013, pp. 239-252.

Müller, Gesine. "La Revue des colonies comme média de transfert au sein d'une diaspora francophone transatlantique." L'Atlantique littéraire: Perspectives théoriques sur la constitution d'un espace translinguistique, edited by Jean Marc Moura and Véronique Porra. Hildesheim: Olms, 2015, pp. 125-142. 
Müller, Gesine. "Las letras: el Caribe francófono e hispanófono en el siglo XIX." Historia comparada de las Antillas, edited by José Antonio Piqueras Arenas. Aranjuez: Ediciones Doce Calles, 2014, pp. 673-706.

Müller, Gesine. "Modelos caribeños de producción teórica: desde la Martinica al Tout-Monde." El caribe como paradigma. Convivencias y coincidencias históricas, culturales y estéticas. Un simposio transareal, edited by Ottmar Ette, Anne Kraume et al. Berlin: tranvía, 2012, pp. 43-57.

Müller, Gesine. "Processes of cultural transfer in 19th-century literature: The Caribbean within the context of the cultural radiance of Europe, exemplified by France and Spain (1789-1886)." Transatlantic Caribbean, edited by Ingrid Kummels, Claudia Rauhut, et al. Bielefeld: transcript, 2014, pp. 239-252.

Müller, Gesine. "Raphaël Confiant: Adèle et la pacotilleuse als Modell karibischer Theorieproduktion." Sondierungen: Lateinamerikanische Literaturen im 21. Jahrhundert, edited by Rike Bolte and Susanne Klengel. Frankfurt am Main, Madrid: Vervuert, Iberoamericana (Series: Biblioteca Ibero-Americana), 2013, pp. 209-221.

Müller, Gesine. "Transkoloniale Dimensionen zwischen Guadeloupe und Kuba: Maryse Condé und Gertrudis Gómez de Avellaneda.” Literatur leben. Festschrift für Ottmar Ette, edited by Albrecht Buschmann, Julian Drews, et al. Madrid: Vervuert, Iberoamericana, 2016, pp. 137-145.

Müller, Gesine. “'Une misérable petite île! moins qu’une île. ...’ Raumdynamiken und koloniale Positionierung in der Literatur der spanischen und französischen Karibik im 19. Jahrhundert." Raum-Bewegung-Passage. Postkoloniale frankophone Literaturen, edited by Gesine Müller and Susanne Stemmler. Tübingen: Narr, 2009, pp. 87-100.

Müller, Gesine. "Variantes de miradas caribeñas hacia constelaciones (pos-)coloniales: Gertrudis Gómez de Avellaneda y Maryse Condé.” Diferencia minoritaria en Latinoamérica / Diferença minoritária na America Latina, edited by Cornelia Sieber, Eduardo Guerreiro Brito Losso, et al. Hildesheim, Zürich, et al.: Olms, 2008, pp. 43-50.

Müller, Gesine. "Victor Hugo y Pierre Loti: los océanos como paradigmas culturales." TransPacífico. Conexiones y convivencias en AsiAméricas. Un simposio transareal, edited by Ottmar Ette, Werner Mackenbach, et al. Berlin: tranvía (Walter Frey), 2013, pp. 159 -178 .

Müller, Gesine. “Vom ‘Genuß der Dinge, die wir so weit herholen ...': Hautfarbe und Zucker bei J. B. Labat und G. Th. Raynal.” Präsenz und Evidenz fremder Dinge im 18. Jahrhundert, edited by Birgit Neumann. Göttingen: Wallstein (Series: 18. Jahrhundert), 2015, pp. 356-372.

Müller, Gesine. "Writing In-Between: Transcultural Positionings of the Free People of Color in Nineteenth-Century Louisiana." New Orleans and the Global South. Caribbean, Creolization, Carnival, edited by Ottmar Ette and Gesine Müller. Hildesheim: Olms, 2017, pp. 115-133.

Müller, Gesine, and Natascha Ueckmann, editors. Kreolisierung revisited. Debatten um ein weltweites Kulturkonzept. Bielefeld: transcript, 2013.

Müller, Gesine, and Natascha Ueckmann. "Einleitung: Kreolisierung als weltweites Kulturmodell?" Kreolisierung revisited. Debatten um ein weltweites Kulturmodell, edited by Gesine Müller and Natascha Ueckmann. Bielefeld: transcript, 2013, pp. 7-42.

Naranjo Orovio, Consuelo. "Los rostros del miedo. El rumor de Haití en Cuba (siglo XIX)." In Caleidoscopios coloniales. Transferencias culturales en el Caribe del siglo XIX = 
Kaléidoscopes coloniaux. Transferts culturels dans les Caraïbes au XIXe siècle, edited by Ottmar Ette and Gesine Müller. Madrid, Frankfurt am Main: Iberoamericana, Vervuert, 2010, pp. 283-304.

O'Brien, Colleen C. Race, Romance, and Rebellion: Literatures of the Americas in the Nineteenth Century. Charlottesville: University of Virginia Press, 2013.

Ogden, Daryl. "Byron, Italy, and the Poetics of Liberal Imperialism." Keats-Shelley Journal, no. 49,2000 , pp. $114-37$.

Osterhammel, Jürgen. The Transformation of the World: A Global History of the Nineteenth Century, translated by Patrick Camiller. Princeton: Princeton University Press, 2015. (Die Verwandlung der Welt. Eine Geschichte des 19. Jahrhunderts. Munich: Beck, 2010.)

Padura Fuentes, Leonardo. José María Heredia. La patria y la vida. Havana: Unión, 2003.

Pagden, Anthony. "Die Auslöschung der Differenz. Der Kolonialismus und die Ursprünge des Nationalismus bei Diderot und Herder." Jenseits des Eurozentrismus. Postkoloniale Perspektiven in den Geschichts- und Kulturwissenschaften, edited by Sebastian Conrad and Shalini Randeira. Frankfurt am Main, New York: Campus, 2002, pp. 116-147.

Pagni, Andrea. "Traducción y transculturación en el siglo XIX. Atala de Chateaubriand por Simón Rodríguez (1801) y el Cancionero de Heine por José A. Pérez Bonalde (1885).” Iberoamericana, vol. 24, nos. 2/3 (78/79), 2000, pp. 88-103.

Painter, Nell Irvin. The History of White People. New York: Norton, 2010.

Pâme, Stella. Cyrille Bissette. Un Martyr de la Liberté. Fort-de-France: Désormeaux, 1999.

Pedraz Marcos, Azucena. Quimeras de África. La sociedad Española de Africanistas y Colonialistas. Madrid: Polifemo, 2000.

Pedraza Jiménez, Felipe B., and Eugenio Alonso Martín. Manual de literatura hispanoamericana. Berriozar, Navarra: Cénlit Ed., 1991.

Piqueras Arenas, José Antonio. La revolución democrática (1868-1874). Cuestión social, colonialismo y grupos de presión. Madrid: Ministerio de Trabajo y Seguridad Social, 1992.

Pons, André. "Blanco White Abolicionista." Cuadernos Hispanoamericanos, no. 559, 1997, pp. 63-76.

Popkin, Jeremy D. Facing Racial Revolution. Eyewitness Accounts of the Haitian Insurrection. Chicago: The University of Chicago Press, 2007.

Poumier, María. "José María Heredia et la révolution française (Cuba 1803-Mexico 1839)." Cahiers des Amériques Latines, no. 10, 1990, pp. 262-274.

Pratt, Mary Louise. Imperial Eyes. Travel Writing and Transculturation. London: Routledge, 1992.

Reinstädler, Janett. "La répétition interrompue. Representando la descentralización del caribe durante la Revolución Francesa." Caribbean(s) on the Move. Archipiélagos literarios del Caribe. A TransArea Symposium, edited by Ottmar Ette. Frankfurt am Main: Peter Lang, 2008, pp. 23-38.

Reinstädler, Janett. "Die Theatralisierung der Karibik. Postkoloniale Inszenierungen auf den spanisch- und französischsprachigen Antillen im 19. Jahrhundert.” Diss. Berlin: Humboldt-Universität, 2006.

Rétat, Pierre. "Citoyen-Sujet, Civisme." Handbuch politisch-sozialer Grundbegriffe in Frankreich 1680-1820, vol. 9. Munich: Oldenbourg, 1988, pp. 75-105. 
Riedel, Wolfgang. "Anthropologie und die Literatur in der deutschen Spätaufklärung.” Internationales Archiv für Sozialgeschichte der deutschen Literatur, special issue \#6, 1994, pp. 93-157.

Rimpau, Laetitia. Reisen zum Ursprung: das Mauritius-Projekt von Jean-Marie Gustave Le Clézio. Tübingen: Niemeyer, 2002.

Rivs, Zelideth Maria, and Debbie Lee-DiStefano, editors. Imagining Asia in the Americas. New Brunswick, New Jersey: Rutgers University Press, 2016.

Roldán, Inés de. "La diplomacia Británica y la abolición del tráfico de esclavos cubanos. Una nueva aportación.” Quinto Centenario, no. 2, 1981, pp. 219-225.

Ronzón, Elena. Antropología y antropologías. Ideas para una historia crítica de la antropología española. Oviedo: Pentalfa, 1991.

Rosa, Richard. Los fantasmas de la razón. Una lectura material de Hostos. San Juan (Puerto Rico), Santo Domingo (Dominican Republic): Isla Negra, 2003.

Rössner, Michael. "Das Bild der Indios in der brasilianischen und hispanoamerikanischen Romantik." Dulce et decorum est philologiam colere. Festschrift für Dietrich

Briesemeister zu seinem 65. Geburtstag, edited by Sybille Große and Axel Schönberger. Berlin: Domus Ed. Europaea, 1999, vol. II, pp. 1709-1726.

Said, Edward W. Culture and Imperialism. New York: Vintage Books, 1994.

Schmeling, Manfred, and Monika Schmitz-Emans. Introduction to Das Paradigma der Landschaft in Moderne und Postmoderne. (Post-)Modernist Terrains: LandescapesSettings-Spaces, edited by Manfred Schmeling and Monika Schmitz-Emans. Würzburg: Königshausen \& Neumann, 2007, pp. 21-36.

Schmidt-Nowara, Christopher. Empire and Antislavery. Spain, Cuba, and Puerto Rico, 1833-1874. Pittsburgh, PA: University of Pittsburgh Press, 1999.

Schmieder, Ulrike. Nach der Sklaverei. Martinique und Kuba im Vergleich. Berlin, Münster: Lit, 2014.

Schulin, Ernst. Die Französische Revolution. Munich: Beck, 1989.

Servera, José. “Introduction.” Sab, by Gertrudis Gómez de Avellaneda, edited by José Servera. Madrid: Cátedra, 1997, pp. 9-93.

Smith, Lynn, and Vernon J. Parenton. "Acculturation among the Louisiana French.” American Journal of Sociology, vol. 44, no. 3, 1938, pp. 355-364.

Sommer, Doris. Foundational Fictions. The National Romances of Latin America. Berkeley: University of California Press, 1991.

Staum, Martin S. Labeling People. French Scholars on Society, Race, and Empire, 1815-1848. Montreal: McGill-Queens University Press, 2003.

Steiner, George. Extraterritorial: Papers on Literature and the Language Revolution. London: Faber and Faber, 1972.

Strubel, Antje Ravic. Die Arbeiter des Meeres. 2003. Available online: http://www.dradio.de/ dlf/sendungen/buechermarkt/165895/. Accessed 21 June 2016.

Tessonneau, Alex-Louise. "Dupré et la littérature jaune en Haïti sous Henri Christophe.” Haïti 1804. Lumières et ténèbres. Impact et résonances d'une révolution, edited by Leon-François Hoffmann, Frauke Gewecke, et al. Madrid: Iberoamericana, 2008, pp. $183-200$.

Thiem, Annegret. Rauminszenierungen. Literarischer Raum in der karibischen Prosaliteratur des 19. Jahrhunderts. Berlin, Münster: Lit, 2010. 
Thoma, Heinz. "Von der Geschichte des esprit humain zum esprit français. Anthropologie, kulturelle Ordnungsvorstellungen und Literaturgeschichtsschreibung in Frankreich 1790-1840." Die Ordnung der Kulturen. Zur Konstruktion ethnischer, nationaler und zivilisatorischer Differenzen 1750-1850, edited by Hansjörg Bay and Kai Merten. Würzburg: Königshausen \& Neumann, 2006, pp. 99-120.

Tietz, Manfred, and Dietrich Briesemeister, editors. Los jesuitas españoles expulsos. Su imagen y su contribución al saber sobre el mundo hispánico en la Europa del siglo XVIII. Proceedings of the International Conference in Berlin, April 7-10, 1999. Frankfurt am Main: Vervuert, 2001.

Tomich, Dale W. Slavery in the Circuit of Sugar. Martinique and the World-Economy, 1830 -1848. Albany: State University of New York Press, 2016.

Torabully, Khal. "Quand les Indes rencontrent les imaginaires du monde." Worldwide. Archipels de la mondialisation. Archipiélagos de la globalización, edited by Ottmar Ette and Gesine Müller. Madrid, Frankfurt am Main: Iberoamericana, Vervuert, 2012, pp. $63-72$.

Torres Cuevas, Eduardo: Antonio Maceo: Las ideas que sostienen el arma. La Habana: Editorial de Ciencias Sociales, 1995.

Torres-Saillant, Silvio. An Intellectual History of the Caribbean. New York: Palgrave Macmillan, 2006.

Toumson, Roger. La transgression des couleurs. Littérature et langage des Antilles, XVIIIe, XIXe, XXe siècles. Paris: Éd. Caribéennes, 1989.

Trouillot, Michel-Rolph. "Zur Bagatellisierung der haitianischen Revolution.” Jenseits des Eurozentrismus. Postkoloniale Perspektiven in den Geschichts- und Kulturwissenschaften, edited by Sebastian Conrad. Frankfurt am Main: Campus, 2002, pp. 84-115.

Turcotte, Paul, and Claude Brabant. "Ile Maurice: Nuvo Sime." Peuples Noirs/Peuples Africains, no. 31, 1983, pp. 100-106.

Vigier, Philippe. "The Reconstruction of the French Abolitionist Movement under the July Monarchy." The Abolitions of Slavery. From Léger Félicité Sonthonax to Victor Schœelcher, 1793, 1794, 1848, edited by Marcel Dorigny. Paris: UNESCO, 2003, pp. 248-254.

Vila Vilar, Enriqueta, and Luisa Vila Vilar, editors. Los abolicionistas españoles. Siglo XIX. Madrid: Ediciones de Cultura Hispánica, 1996.

“Völker am Wasser." http://www.wunderhorn.de/content/buecher/literarische_reihen/voelk er_am_wasser/index_ger.html. Accessed June 232016.

Walker, David. Walker's Appeal, in Four Articles; Together with a Preamble, to the Coloured Citizens of the World, but in Particular, and Very Expressly, to Those of the United States of America, Written in Boston, State of Massachusetts, September 28, 1829. Boston, 1830; electronic edition available at Documenting the American South (DocSouth). University of North Carolina at Chapel Hill. http://docsouth.unc.edu/nc/walker/walker. html. Accessed June 232016.

Walker, David. David Walker's Appeal, In Four Articles: Together With A Preamble To The Coloured Citizens Of The World, But In Particular, And Very Expressly, To Those Of The United States Of America, revised edition with an introduction by Sean Wilentz. New York: Hill and Wang, 1995; excerpts online available at http://www.pbs.org/wgbh/aia/ part4/4 h2931 t.html. Accessed June 232016. 
Walsh, John Patrick. Free and French in the Caribbean: Toussaint Louverture, Aimé Césaire, and Narratives of Loyal Opposition. Bloomington, Indiana: Indiana University Press; Chesham: Combined Academic, 2013.

Warning, Rainer. "Romantische Tiefenperspektivik und moderner Perspektivismus. Chateaubriand/Flaubert/Proust." Romantik. Aufbruch zur Moderne, edited by Karl Maurer and Winfried Wehle. Munich: Fink, 1991, pp. 295-324.

Watson, Tim. Caribbean Culture and British Fiction in the Atlantic World, 1780-1870. Cambridge: Cambridge University Press, 2008.

Weigel, Sigrid. 'Zum 'topographical turn.' Kartographie, Topographie und Raumkonzepte in den Kulturwissenschaften.” KulturPoetik, vol. 2, no. 2, 2002, pp. 151-165.

Werner, Michael, and Bénédicte Zimmermann. "Vergleich, Transfer, Verflechtung. Der Ansatz der 'Histoire croisée' und die Herausforderung des Transnationalen." Geschichte und Gesellschaft. Zeitschrift für historische Sozialwissenschaft, no. 28, 2002, pp. 607-636.

Wogatzke, Gudrun. Identitätsentwürfe. Selbst- und Fremdbilder in der spanisch- und französischsprachigen Prosa der Antillen im 19. Jahrhundert. Frankfurt am Main: Vervuert, 2006.

Wogatzke-Luckow, Gudrun. “Victor Hugo: Bug-Jargal (1826). Abgesang auf den 'bon sauvage' oder Inszenierung von Ambivalenzen?" Pasajes. Homenaje a Christian Wentzlaff-Eggebert = Passages. Mélanges offerts à Christian Wentzlaff-Eggebert = Passagen. Festschrift für Christian Wentzlaff-Eggebert, edited by Susanne Grunwald. Sevilla: Secretariado de Publicaciones de la Universidad de Sevilla, 2004, pp. 21-138.

Wolfzettel, Friedrich. "Ce désir de vagabondage cosmopolite." Wege und Entwicklung des französischen Reiseberichts im 19. Jahrhundert. Tübingen: Niemeyer, 1986.

Zacair, Philippe. "Haiti on His Mind. Antonio Maceo and Caribbeanness." Caribbean Studies, no. 33, 2005, pp. 47-78.

Zeuske, Michael. “Gran Caribe.” http://www.ihila.uni-koeln.de/5593.html. Accessed 5 December 2010; site discontinued.

Zeuske, Michael. Kleine Geschichte Kubas. Munich: Beck, 2000.

Zeuske, Michael. Schwarze Karibik. Sklaven, Sklavereikultur und Emanzipation. Zurich: Rotpunktverlag, 2004.

Zeuske, Michael. Sklavenhändler, Negreros und Atlantikkreolen. Berlin, Boston: De Gruyter Oldenbourg, 2015. 